\title{
Analgesic nephropathy and transitional cell carcinoma
}

\author{
V. MoshaKIS \\ F.R.C.S. \\ A. A. HOOPER \\ F.R.C.S. \\ Queen Mary's Hospital for the East End, West Ham Lane, Stratford, London E15 4SD
}

\begin{abstract}
Summary
A case of transitional cell carcinoma of the kidney associated with long-term phenacetin intake is described and the practical implications discussed.
\end{abstract}

\section{Introduction}

Cancer of the bladder was first described from aniline workers in 1895 . Since then various compounds have been proved to be aetiologically connected with transitional cell carcinoma of the urothelium including $\alpha$ - and $\beta$-naphthylamine, benzidine and benzidine homologues such as orthotolidine and 4-aminodiphenyl. Cigarette smoking and Balkan nephropathy are now accepted as environmental factors. A further case of transitional cell carcinoma of the urothelium is emerging in the form of analgesic nephropathy. The association has been recognized and described mainly in Scandinavia (Rathert, Melchior and Lutzeyer, 1975; Angervall et al., 1969).

Reviewing the world literature, this is the first case reported from England.

\section{Case report}

A 42-year-old woman presented with a seven-week history of right-sided abdominal pain, vomiting, anorexia and weight-loss. Three years previously, in the course of investigations for headaches, she was found to have taken an average of six Codeine Compound tablets daily since the age of thirteen for dysmenorrhoea, headaches and attacks of 'cystitis'. This represents a total of $14 \mathrm{~kg}$ of phenacetin over 26 years. On examination she was found to have a palpable and tender right kidney.

An i.v.p. showed that the left kidney excreted promptly but it was small and had an abnormal calyceal pattern consistent with analgesic nephropathy. On the right side there was no evidence of function although the two hour film showed a possible nephrogram. Other relevant investigations were as follows: urine analysis, presence of red cells ++ ; chest X-ray, no abnormality present; blood urea, $5.0 \mathrm{mmol} / 1$; MSU, no growth; $\mathrm{Hb}, 11.6 \mathrm{~g} / \mathrm{dl}$; WBC, $15.9 \times 10^{9} / 1$; neutrophils, $84 \%$, lymphocytes $13 \%$; ESR, $95 \mathrm{~mm} / \mathrm{hr}$.
At cystoscopy, tumour was seen protruding from the right ureteric orifice and an ascending ureterogram showed multiple filling defects in the ureter and non-filling of the renal pelvis.

Accordingly, nephro-ureterectomy was undertaken. The kidney and ureter were found to be involved extensively with tumour. There was considerable spread of the disease to the local lymph nodes. Microscopy revealed a very anaplastic and widely invasive transitional cell carcinoma. The renal papillae were completely destroyed by tumour and therefore it was impossible to demonstrate histological evidence of papillary necrosis.

\section{Discussion}

The carcinogenic effect of phenacetin could be explained by the fact that it is an aromatic amide and studying the chemistry of the drug has revealed that its metabolic pathway is similar to the proved carcinogen $\beta$-naphthylamine. Both compounds produce similar $O$-hydroxyamino metabolites (Leading Article, 1969).

Although occasional cases have been described in other countries, most reports of this association come from Sweden. Johannson (1974) has postulated that this may represent the effect of early withdrawal of phenacetin (in 1961) from overthe-counter sales in Sweden, unlike other countries where phenacetin was prescribed until much later. Thus, in countries other than Sweden, patients with analgesic renal damage died from uraemia before the tumour had time to appear.

Analgesic nephropathy is being recognized with increasing frequency in the U.K. Therefore, it is important to emphasize that urological symptoms in patients with known analgesic abuse or proved analgesic renal damage, may indicate the development of transitional cell carcinoma. Such patients merit full urological investigations, especially if there is haematuria, since this finding is not commonly seen in analgesic nephropathy. This case reinforces the view of previous writers (Rathert et al., 1975) that exfoliative urinary cytology at regular intervals is imperative in patients with suspected or known analgesic papillary necrosis. Similarly, a full drug 
history should be obtained from patients who have undergone nephro-ureterectomy for transitional cell carcinoma of the ureter or kidney, since arresting the damage to the remaining kidney is of paramount importance.

The incidence of carcinoma of the urothelium in this country is increasing and it is possible that phenacetin may be partly responsible. Since the mechanism of tumour production by phenacetin is likely to be the same as the other aromatic amides, one can expect the latent period before a tumour appears to be similar to that from aromatic amides. It is therefore possible that there will be a further increase of transitional cell carcinoma of the uro- thelium, even if other aetiological factors are not considered.

\section{References}

Angervall, L., Bengtsson, H., Zutterland, C.G. \& ZsigmoD, M. (1969) Renal pelvic carcinoma in a Swedish district with abuse of a phenacetin containing drug. British Journal of Urology, 41, 401.

JohANSSON, S. (1974) Uro-epithelial tumors of the renal pelvis associated with abuse of phenacetin containing analgesics. Cancer. New York, Philadelphia, etc., 33, 743.

LEADING ARTICLE (1969) Analgesic abuse and tumours of the renal pelvis. Lancet, ii, 1233.

Rathert, P., Melchior, H., Lutzeyer, W. (1975) Phenacetin - a carcinogen for the urinary tract. Journal of Urology, 113, 653 .

\title{
Partial lipodystrophy with nephrotic syndrome
}

\author{
A. M. HUNTER* $\ddagger$ \\ M.B.Ch.B., M.R.C.P. \\ A. A. H. LAWSON* \\ M.D., F.R.C.P.E. \\ D. THOMSON $\dagger$ \\ M.B.Ch.B., M.R.C.Path. \\ *Milesmark Hospital, Dunfermline, and †Department of Pathology, University New Buildings, \\ University of Edinburgh
}

\begin{abstract}
Summary
A patient with nephrotic syndrome in association with partial lipodystrophy is reported. The features of partial lipodystrophy are well recognized and the renal lesion is a mesangiocapillary glomerulo-nephritis of a dense deposit type with an associated depression of C3. This type of kidney involvement is becoming increasingly recognized as common in the syndrome of partial lipodystrophy.

\section{Introduction}

Partial lipodystrophy is a rare syndrome which has been defined as 'symmetrical loss of fat from the face with or without disappearance of fat from the arms, chest, abdomen and hips but with normal distribution on the lower extremities' (Nelson, Vaughan and MacKay, 1969). The aetiology is unknown but an infective illness is present often at the onset (Poley and Stickler, 1963). The syndrome

$\ddagger$ Present address: Registrar in Chest Diseases, City Hospital, Edinburgh.
\end{abstract}

is commoner in females (four females : one male) and usually presents at between 5 and 15 years of age. The most extensive review of the literature is that of Senior and Gellis (1964) in which 77 cases were reported. A renal disorder occurred in $20 \%$ of these patients and a wide variety of types were noted (pyelonephritis, acute and chronic glomerulonephritis, calyceal dilatation, hydronephrosis, haematuria, asymptomatic proteinuria and the nephrotic syndrome). Other associated abnormalities were - central nervous system disturbance; hepatomegaly; hyperlipaemia; abnormal glucose tolerance tests and one case of diabetes mellitus, as well as single reports of abnormal pigmentation, hirsutism and episodic diarrhoea.

Recent interest has been in the associated renal lesions. The purpose of this communication is to describe a patient with the nephrotic syndrome in association with partial lipodystrophy, in whom renal biopsy studies were performed with immunofluorescent and electron microscopical findings. 